\title{
Recurrent Spontaneous Coronary Artery Dissection Leading to Recurrent Sudden Cardiac Arrests: Who is at risk?
}

\author{
Anthony Weber ${ }^{1}$, Joseph Elliott ${ }^{1}$, and Prabhakaran Gopalakrishnan ${ }^{2}$ \\ ${ }^{1}$ Canton Medical Education Foundation \\ ${ }^{2}$ Aultman Hospital
}

May 6, 2020

\begin{abstract}
Spontaneous coronary artery dissection(SCAD) is a rare cause of acute coronary syndrome(ACS) with medical management and conservative therapies usually resulting in favorable outcomes. SCAD often is recurrent especially in high risk individuals with underlying connective tissue disorders. Lethal ventricular arrhythmias have been reported in SCAD without clear indications for secondary prevention with implantable cardiovert-defibrillator(ICD). Depending on risk factor for recurrence as well as susceptibility towards ventricular arrhythmias some patients may benefit from early implantations of ICDs.
\end{abstract}

\section{Introduction:}

Spontaneous Coronary Artery Dissection (SCAD) is defined as an epicardial coronary artery dissection not associated with atherosclerosis, trauma or iatrogenic causes ${ }^{1,2}$. SCAD is a rare cause of acute coronary syndrome(ACS) that can affect single or multiple coronary vessels ${ }^{3,4}$. Complications of SCAD include ongoing ischemia, cardiomyopathy, arrhythmias and sudden cardiac death ${ }^{3,4}$. Coronary angiography is the primarily imaging modality for diagnosing $\mathrm{SCAD}^{4}$. Medical management and conservative therapy for SCAD typically results in favorable outcomes ${ }^{5}$, but there is a high recurrence rate of major cardiovascular events with the majority being $\mathrm{SCAD}^{2,6}$. Major adverse cardiovascular events (MACE) can range from recurrent MI, recurrent SCAD, unanticipated revascularization, congestive heart failure, stroke, and death. To date, there are no definite primary or secondary preventative measures for either SCAD or the associated cardiovascular complications. Our case presents a patient with an underlying connective tissue disorder who developed recurrent SCAD in multiple coronary arteries. This resulted in recurrent episodes of ventricular fibrillation and cardiac arrest despite optimized medical therapy. We discuss the role of implantable cardiovert-defibrillator (ICD) for secondary prevention in patients with recurrent SCAD.

\section{Case presentation:}

A 59-year-old female with history of hypertension, hyperlipidemia and undifferentiated connective tissue disorder on chronic steroids presented in 2012 for intermittent chest pain following emotional stress. Her initial workup showed new onset left bundle branch block and an elevated high sensitivity troponin. She underwent urgent cardiac catheterization, revealing $100 \%$ occlusion of the first obtuse marginal branch (Figure 1, left) with attempted, but unsuccessful percutaneous coronary intervention (PCI). Left ventriculogram showed ejection fraction (EF) of $40 \%$ and severe distal inferior and inferoapical hypokinesis. The wall motion abnormalities were not explained by the coronary anatomy and were attributed to possible concomitant Takotsubo's cardiomyopathy in the setting of acute myocardial infarction. Patient was treated medically.

Three years later in 2015, the patient presented for recurrent chest discomfort following the death of a family member. While being evaluated in the emergency room, she developed ventricular fibrillation arrest. She underwent defibrillation and was successfully resuscitated. Urgent cardiac catheterization was performed 
revealing a healed obtuse marginal branch (figure 1, right). However, spontaneous dissection of the small caliber first diagonal branch was visualized (figure 2, left). Echocardiogram revealed an ejection fraction 35-40\%. Cardiac Electrophysiologist was consulted for possible ICD placement for secondary prevention of sudden cardiac arrest (SCA). Given her acute ischemic event was within 48 hours of her arrest, patient was treated medically with beta blockers.

The patient returned 2 years later in 2017 at the age of 64 , again with substernal chest pain. On arrival, initial high sensitivity troponin was 10.3. Within thirty minutes, she suffered a ventricular fibrillation arrest. She underwent defibrillation and was successfully resuscitated. Emergent cardiac catheterization showed improved first diagonal disease when compared to 2015(figure 2, right) but the left anterior descending artery (LAD) showed severe diffuse distal narrowing (figure 3, left) compared to coronary angiogram in 2015 (Figure 3, middle) suggesting SCAD. Patient remained hemodynamically stable without signs of ongoing ischemia. Given that this was the third occurrence of SCAD, she underwent CT angiography of the head and abdomen. Findings were suggestive of fibromuscular dysplasia involving bilateral carotid and renal arteries. On the third day of the hospital course, she developed recurrent chest pain, with repeat high-sensitivity troponin greater than $40 \mathrm{ng} / \mathrm{dl}$. Cardiac catheterization was again performed, with a worsening subtotal occlusion of the distal LAD with proximal extension of the dissection (figure 3, right). Echocardiogram was performed that showed ejection fraction to be $45 \%$ without regional wall motion abnormalities. Her symptoms eventually resolved, she remained stable and was treated conservatively. Patient did not meet standard criteria for ICD placement as both her episodes of SCA were within 48 hours of an ischemic event. Regardless, because of her propensity for recurrent SCAD as well as two prior episodes of SCA, the patient was discharged home an external cardioverter-defibrillator and eventually underwent an ICD placement as outpatient.

\section{Discussion:}

Spontaneous coronary artery dissection is an important cause of ACS that is increasingly recognized with use of cardiac imaging modalities. Reported risk factors include connective tissue disorders, fibromuscular dysplasia, as well as chronic inflammatory disorders ${ }^{1,3,5}$. SCAD has also been associated with extreme emotional or physical triggers much like Takotsubo's cardiomyopathy ${ }^{1,3}$. It has a predilection for young, otherwise healthy middle-age women without any traditional cardiovascular risk factors. Although it is quite rare amongst the general population, in a select population of young women who present with ACS, SCAD accounted for up to $24 \%$ of cases $^{3}$.

There are documented cases of SCAD occurring in different age groups ranging from the second to eighth decade of life. With increasing awareness of this once elusive entity, it is estimated that SCAD may cause from $0.1 \%-4 \%$ of ACS cases and LAD appears to be the most commonly affected vessel ${ }^{1,3,5}$. These patients are at high risk for recurrence of MACE. Major cardiac events within 5-7 years are reported at 15-37\% and at 10 years was estimated to be approximately $50 \%$. The majority of these events were related to recurrent $\mathrm{SCAD}^{1,6,7}$.

Despite its similarity to atherosclerotic ACS in clinical presentation as well as propensity for coronary ischemia, PCI should not be routinely performed because of the increased risk of complications and limited success $^{1,2,4}$. In addition, the majority of SCAD lesions spontaneously resolve without any intervention ${ }^{1,4,5}$. Therefore, conservative therapy is recommended over PCI for stable patients without ongoing ischemia. For patients with high-risk lesions or hemodynamic instability, PCI and CABG have been performed, but trials comparing outcomes of conservative versus have revascularization outcomes are lacking ${ }^{1,4,5}$.

Outcomes of SCAD can range from complete resolution to infarction, cardiomyopathy, ventricular arrhythmias and sudden cardiac death. Saw et al. published a cohort study in 2019 of 750 patients who presented with SCAD with $8.1 \%$ presenting with ventricular arrhythmias. The rate of MACE at 30 days was quite high at $8.8 \%$ although mortality during this time period was less than $1 \%^{7}$. This study had short follow up and did not define patients at highest risk of lethal arrhythmias and sudden cardiac death. Longer follow up and risk stratification is needed to clearly identify the at-risk population. 
Literature review shows, $3-11 \%$ of SCAD patients present with ventricular arrhythmias or sudden cardiac death $^{1,2}$. In this case report, patient initially received wearable external defibrillator and subsequently underwent ICD placement, due to her recurrent sudden cardiac arrest(SCA) caused by SCAD. While individual cases of ICD implantation for secondary prophylaxis for SCA in SCAD have been reported, there is no consensus on timing or criteria for ICD placement in these patients. The risk of SCA was higher in patient with tobacco abuse, ST-segment elevation at presentation, pregnancy status and recurrent $\mathrm{SCAD}^{8}$. A retrospective study by Lane et al., found that multivessel SCAD had more than twice the incidence of SCD (34\% vs 16\%) compared to single vessel SCAD but did not show any benefit for external or internal defibrillator placement in patients with SCAD and SCD ${ }^{9}$. There are no existing guidelines to identify SCAD patients at highest risk of ventricular arrhythmias and sudden cardiac death. Prospective or large retrospective studies looking at SCD risk in SCAD as well as role of ICD in SCAD patients who present with SCD, are lacking. The risks and benefits of this invasive procedure should be further evaluated with long term follow up to establish practice standards. Our patient likely represents a sub group with high risk for both SCAD as well as SCD and may have been a good candidate for ICD placement following her first cardiac arrest. This patient was fortunate enough to suffer her lethal arrhythmias inside the hospital, as out of hospital cardiac arrest have lower likelihood of survival to discharge compared to in hospital cardiac arrest (10-12\% vs $25 \%)^{10}$. Studies are needed to identify SCAD patients who have higher risk for recurrence of SCAD as well as SCD.

\section{Conclusion:}

Life threatening arrhythmias is one of the complications of SCAD. Conservative management is recommended for most patients with SCAD and revascularization is typically reserved for high risk patients with life threatening arrhythmias and ongoing ischemia. While consensus exists on revascularization in patients with life threatening arrhythmias, timing and criteria for device therapy for secondary prophylaxis for SCD in the setting of SCAD is unclear. This case highlights the propensity of some patients to have recurrences of SCAD as well as recurrences of life threatening arrhythmias, who would benefit from placement of an implantable cardioverter- defibrillator. Larger studies are needed to identify high risk patients and to define the role of early device therapy in patients with recurrent SCAD with or without life threatening arrhythmias.

\section{References:}

1. Hayes SN, Kim ESH, Saw J, et al. Spontaneous Coronary Artery Dissection: Current State of the Science: A Scientific Statement from the American Heart Association. Circulation. 2018

2. Saw J, Mancini GB, Humphries KH. Contemporary Review on Spontaneous Coronary Artery Dissection. J Am Coll Cardiol. 2016;68(3):297-312.

3. Saw J, Aymong E, Sedlak T, et al. Spontaneous coronary artery dissection: association with predisposing arteriopathies and precipitating stressors and cardiovascular outcomes. Circ Cardiovasc Interv. 2014;7(5):645-55.

4. Tweet MS, Hayes SN, Pitta SR, et al. Clinical features, management, and prognosis of spontaneous coronary artery dissection. Circulation. 2012;126(5):579-88.

5. Alfonso F, Bastante T, García-guimaraes M, et al. Spontaneous coronary artery dissection: new insights into diagnosis and treatment. Coron Artery Dis. 2016;27(8):696-706.

6. Sharma S, Rozen G, Duran J, Mela T, Wood MJ. Sudden Cardiac Death in Patients with Spontaneous Coronary Artery Dissection. J Am Coll Cardiol. 2017;70(1):114-115.

7. Saw J, Straovoytov A, et al. Canadian spontaneous coronary artery dissection cohort study: in-hospital and 30-day outcomes. Euro Heart J, 2019, 40:1188-97

8. Riccardo Scagliola, Claudio Brunelli, et al. Implantable cardioverter defibrillator therapy for secondary prevention in spontaneous coronary artery dissection: to place or not to place? This is the matter. Anatol J Cardiol, 2020;23:240-2

9. Lane C, Tweet M, et al. Incidence of Sudden Cardiac Death in Spontaneous Coronary Artery Dissection and the Role for Implantable Cardiac Defibrillator, J Am Coll Cardiol, 2019 March 12; 73(9)

10. Andersen LW, Holmberg MJ, Berg KM, Donnino MW, Granfeldt A. In-Hospital Cardiac Arrest: A 
Review. JAMA. 2019;321(12):1200-121

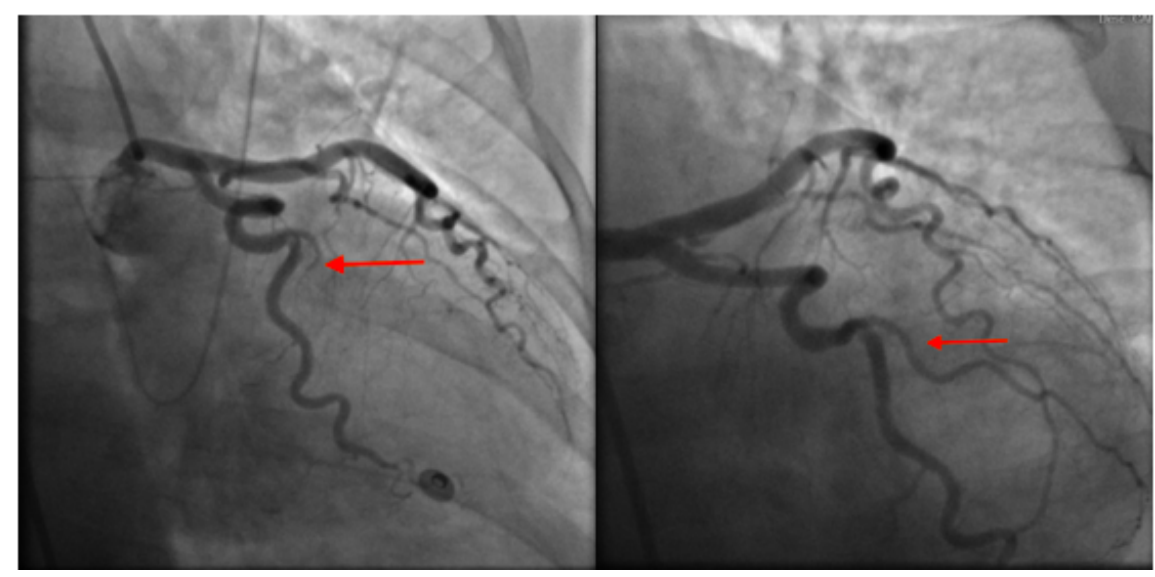

Figure 1: Complete occlusion of the first obtuse marginal branch, as shown on the left, in 2013. Complete resolution of SCAD in the obtuse marginal branch by coronary angiography in 2015 .

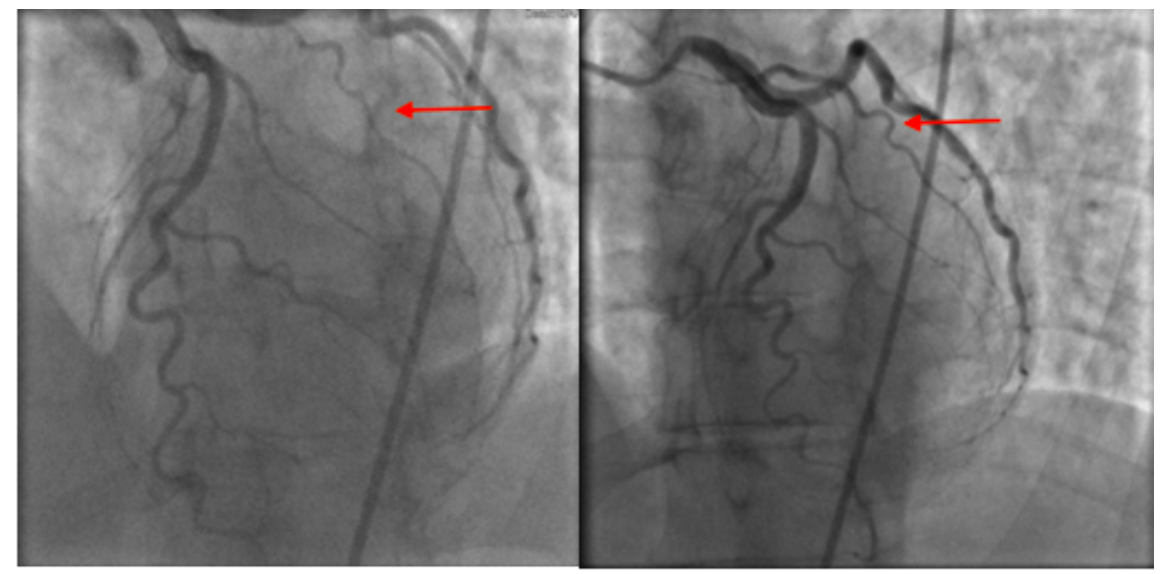

Figure 2: Acute dissection of the first diagonal branch, as seen on the left, in 2015. The normal vessel is shown on right during coronary angiography in 2013.

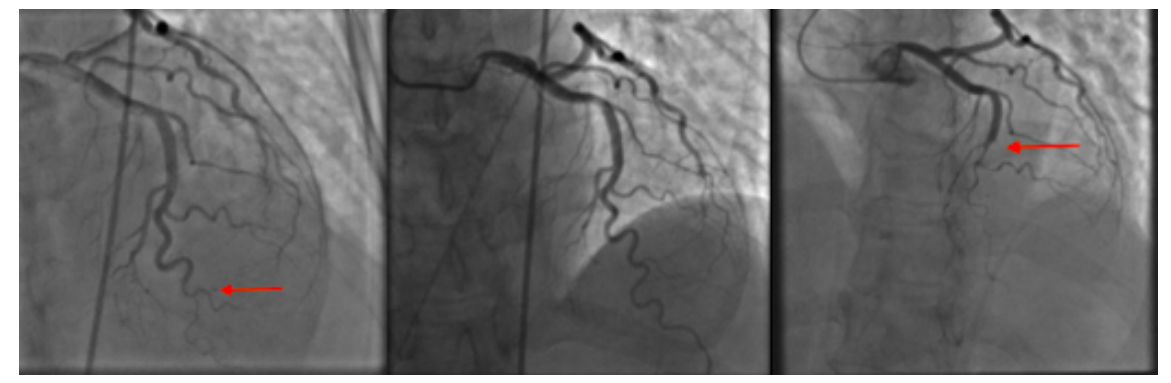

Figure 3: On the left, shows the LAD dissection during coronary angiography in 2017 in comparison to the normal LAD, as seen in the middle panel during 2015. On the right, shows extension of the LAD dissection in 2017. 